\title{
An Autopsy Case of Two Distinct, Acquired Drug Resistance Mechanisms in Epidermal Growth Factor Receptor-mutant Lung Adenocarcinoma: Small Cell Carcinoma Transformation and Epidermal Growth Factor Receptor T790M Mutation
}

\author{
Makoto Furugen ${ }^{1}$, Kayoko Uechi ${ }^{1}$, Jun Hirai ${ }^{1}$, Hajime Aoyama ${ }^{2}$, Masanao Saio ${ }^{2}$, \\ Naoki Yoshimi ${ }^{2}$, Takeshi Kinjo ${ }^{1}$, Kazuya Miyagi ${ }^{1}$, Shusaku Haranaga ${ }^{1}$, Futoshi Higa ${ }^{1}$, \\ Masao Tateyama ${ }^{1}$ and Jiro Fujita ${ }^{1}$
}

\begin{abstract}
We herein describe the case of a 63-year-old man who died from relapsed epidermal growth factor receptor gene $(E G F R)$ exon 19 deletion lung adenocarcinoma treated with erlotinib. According to the autopsy results, he was confirmed to have small cell carcinoma without the EGFR T790M mutation in his pancreas and left kidney metastatic specimens, while the adenocarcinoma metastatic lesion in his right kidney had the EGFR T790M mutation; both retained the somatic EGFR exon 19 deletion. We herein report an autopsy case of resistance to an EGFR tyrosine kinase inhibitor via small cell carcinoma transformation and the EGFR T790M mutation in separate metastatic organs.
\end{abstract}

Key words: $E G F R$ mutation, lung adenocarcinoma, EGFR-TKI, small cell carcinoma transformation, EGFR T790M mutation

(Intern Med 54: 2491-2496, 2015)

(DOI: 10.2169/internalmedicine.54.5481)

\section{Introduction}

Epidermal growth factor receptor tyrosine kinase inhibitors (EGFR-TKIs) are highly effective for patients with EGFR mutant lung adenocarcinoma $(1,2)$. However, most patients ultimately develop acquired resistance, several mechanisms of which have been identified. With respect to the mechanisms of acquired drug resistance, the EGFR T790M mutation $(3,4)$ or $M E T$ gene amplification $(5,6)$ occur frequently, whereas transformation to small cell carcinoma is rarely reported (7-9).

We herein report an autopsy case of acquired resistance to EGFR-TKI therapy through the transformation to small cell carcinoma and a separate EGFR T790M mutation, both of which retained the activating EGFR mutation.

\section{Case Report}

A 63-year-old, non-smoking Japanese man who had palsy of the right side of his face and a slight cough for one month was referred to our hospital because a lung mass lesion was identified on a chest X-ray. Radiological examinations revealed a dominant left upper lung tumor (Fig. 1A), in addition to liver, brain, and bone metastases. The laboratory data showed an elevation of tumor makers [carcinoembryonic antigen (CEA) 13,160 ng/mL, cytokeratin 19 fragment (CYFRA) $81 \mathrm{ng} / \mathrm{mL}$, and squamous cell carcinoma re-

\footnotetext{
${ }^{1}$ Department of Infectious, Respiratory, and Digestive Medicine, Control and Prevention of Infectious Diseases (The First Department of Internal Medicine), Faculty of Medicine, University of the Ryukyus, Japan and ${ }^{2}$ Department of Pathology and Oncology, Graduate School of Medicine, University of the Ryukyus, Japan

Received for publication March 30, 2015; Accepted for publication May 13, 2015

Correspondence to Dr. Makoto Furugen, makoto.furugen@live.jp
} 

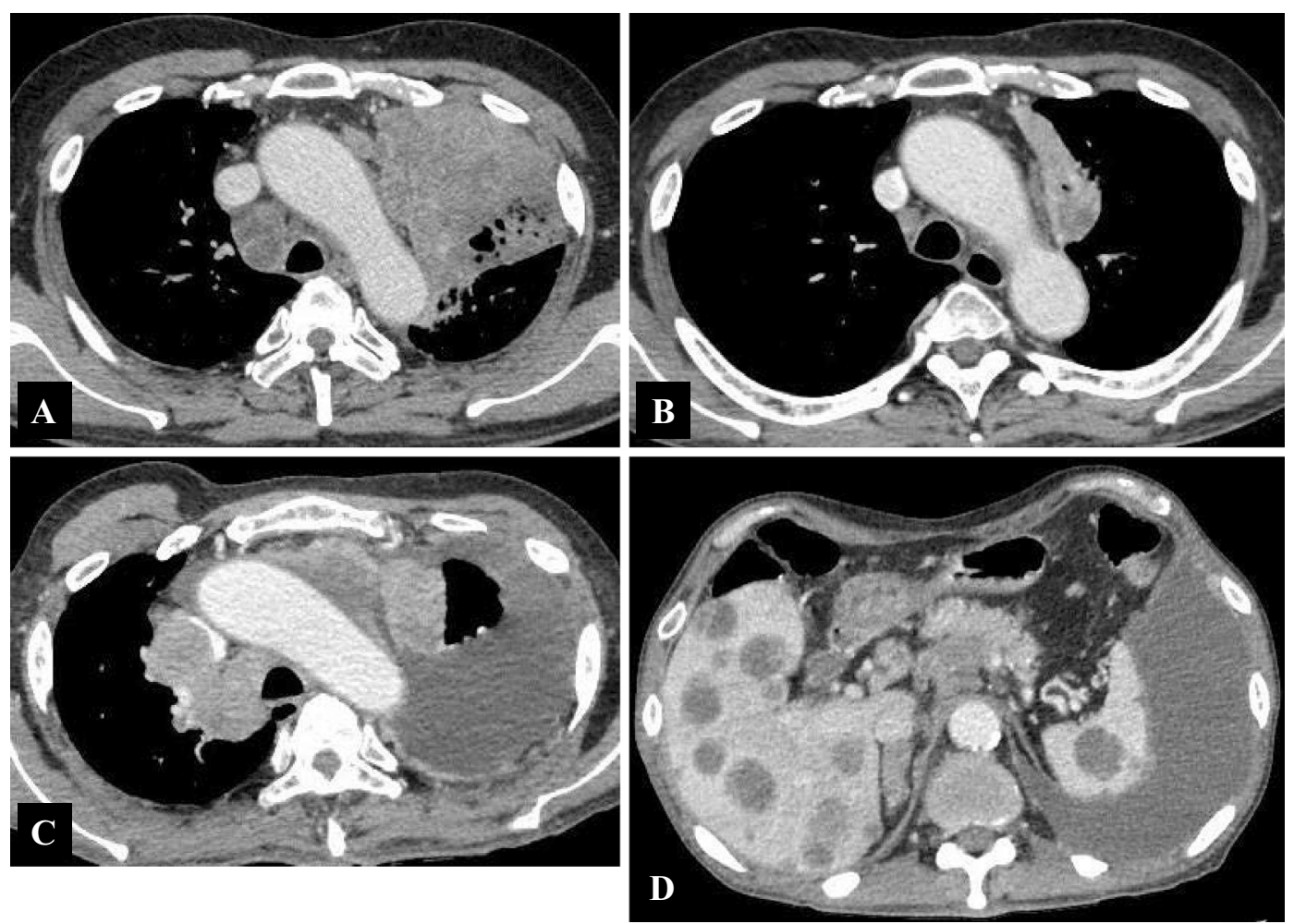

Figure 1. A chest computed tomography scan at the time of diagnosis showing a tumor in the left upper lung lobe and swollen mediastinal lymph nodes (A). After three months of erlotinib treatment, the tumors remarkably improved (B). After approximately 10 months of treatment, the thoracic tumor lesions (C) and multiple liver metastases (D) were enlarged.

lated antigen (SCC) $8.0 \mathrm{ng} / \mathrm{mL}]$. A Trans-bronchial examination revealed stage IV (T4N3M1b) lung adenocarcinoma (Fig. 2) with an EGFR exon 19 deletion (using the cycleave method). He was started on EGFR-TKI (erlotinib: $150 \mathrm{mg}$ per day for the first 18 days, then reduced to $100 \mathrm{mg}$ per day because of liver dysfunction), followed by 30 Gy whole-brain irradiation. Two weeks later, his chest X-ray findings began to improve, and his response was subsequently evaluated as a partial response via computed tomography (CT) scan findings (Fig. 1B). Brain and bone metastases were confirmed to have improved via magnetic resonance imaging scan findings. Because of the treatment, his performance status improved from 3 to 1 , and he continued treatment in an ambulatory setting.

Approximately 10 months later, the patient was readmitted to our hospital because of increased left pleural effusion and enlarged mediastinal lymph nodes. CT showed that the primary lesion in his left upper lung lobe (Fig. 1C) and multiple liver metastases (Fig. 1D) were enlarged, and he was subsequently diagnosed with progressive disease. An examination of the left pleural effusion showed exudative effusion and revealed a mixture of adenocarcinoma and small cell carcinoma cells on cytology. The laboratory data showed a significant elevation of CEA $(7,280 \mathrm{ng} / \mathrm{mL})$, and additionally pro-gastrin-releasing peptide (ProGRP, 145 pg/ $\mathrm{mL}$, which was not detected at the time of diagnosis) and neuron-specific enolase (NSE, 1,410 ng/mL). Additionally, using a gene mutation analysis of the left pleural effusion, carcinoma cells were confirmed to retain a somatic EGFR exon 19 deletion [using the Scorpion amplification refractory mutation system (ARMS) method]. The patient then experienced a decreased level of consciousness and had difficulty in swallowing. Because his general status was rapidly worsening, he was given supportive care without any additional chemotherapy. He died 11 months after the beginning of treatment. We conducted an autopsy with his family's approval.

The autopsy findings revealed that he had multiple systemic metastases, including in the bilateral lungs and mediastinal lymph nodes, liver, pancreas, and adrenal gland metastases. The primary lung lesion consisted of adenocarcinoma and small cell carcinoma (Fig. 3), with limited squamous cell carcinoma. The spleen, right kidney, and spondylus metastatic lesions consisted only of adenocarcinoma cells, whereas the other organ metastatic lesions consisted of only small cell carcinoma (Table). In addition, we conducted a gene mutation analysis from the pancreas and left kidney specimens (small cell carcinoma; Fig. 4A, B), and the right kidney specimen (adenocarcinoma; Fig. 4C), which were first discovered at autopsy. The results revealed that the pancreas and left kidney specimens (small cell carcinoma) were confirmed to have retained the somatic EGFR exon 19 deletion without an EGFR T790M mutation (using the Scorpion ARMS method), whereas the right kidney specimen (adenocarcinoma) was confirmed to have retained the somatic EGFR exon 19 deletion with the EGFR T790M 


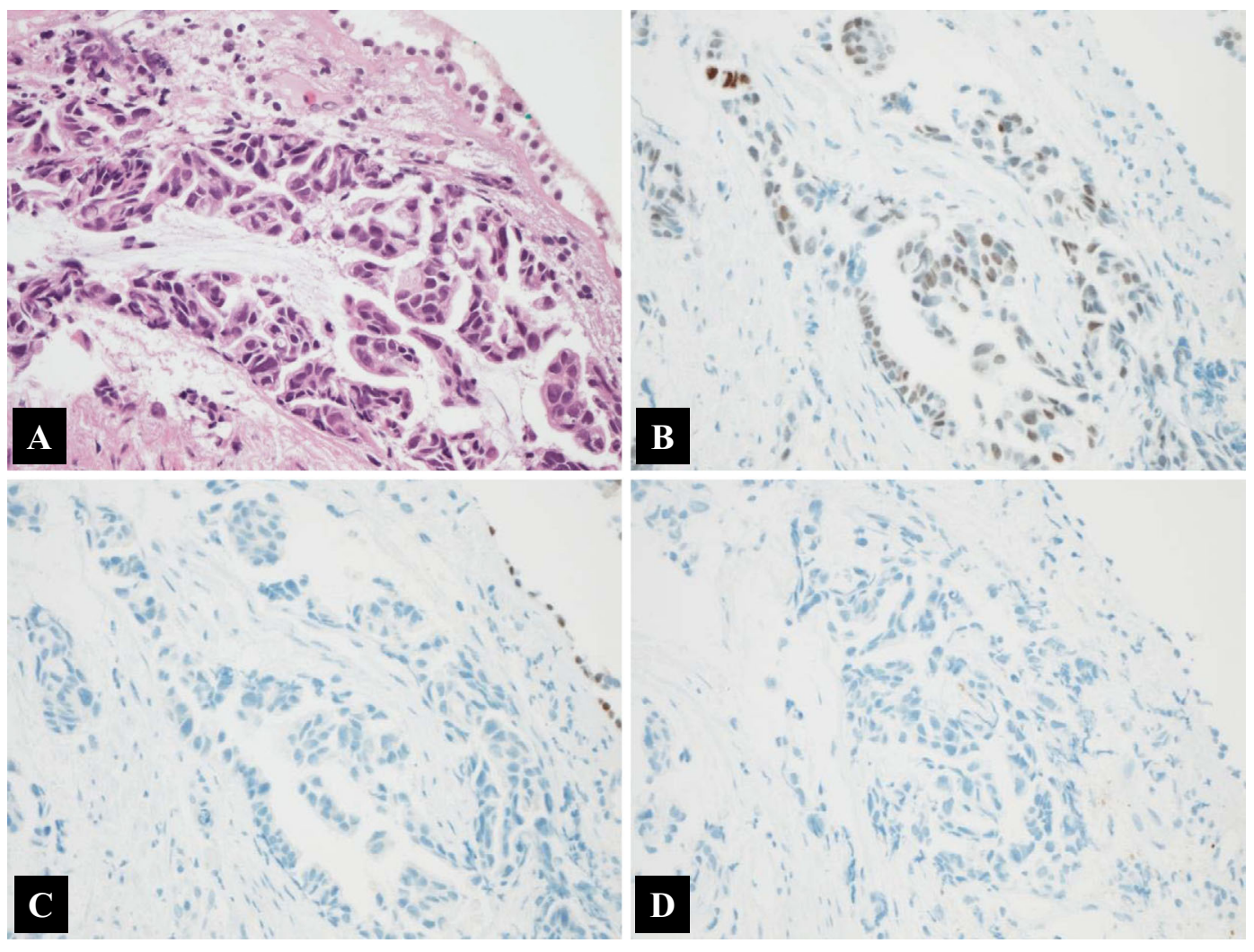

Figure 2. A trans-bronchial biopsy specimen shows poorly differentiated adenocarcinoma (A: Hematoxylin and Eosin staining, 20x original magnification), with positive staining for TTF-1 (B: 20x original magnification). Staining for $\mathbf{p 4 0}$ (a lung squamous cell carcinoma marker) (C: $20 \times$ original magnification) and CD56 (small cell carcinoma marker) (D: 20× original magnification) was negative.

mutation (using the Scorpion ARMS method).

\section{Discussion}

Predominant acquired resistance mechanisms of lung adenocarcinoma to EGFR-TKI therapy have been previously described and include secondary EGFR mutations and the activation of collateral EGFR signaling pathways. With respect to the secondary EGFR mutation, the EGFR T790M mutation is well known and develops in approximately half of lung adenocarcinoma patients with acquired resistance to EGFR-TKI therapy $(3,4)$. In addition, as a collateral pathway in EGFR signaling, MET gene amplification $(5,6)$ and MET activation by its ligand, hepatocyte growth factor (10), are also well established. However, more recently, the uncommon adenocarcinoma to small cell carcinoma transformation in patients with a somatic activating EGFR mutation as a resistant mechanism to EGFR-TKI therapy was reported by Sequist et al. (8).

In the present case, small cell carcinoma was detected from a primary lung adenocarcinoma patient with a somaticactivating EGFR mutation while taking EGFR-TKI therapy (erlotinib). We suspected that small cell carcinoma constituted part of the tumor at the first diagnosis or that a secondary carcinoma had occurred during the clinical course. However, we diagnosed the lesion to be small cell carcinoma transformation from lung adenocarcinoma for the fol- lowing three reasons: 1) small cell carcinoma was not detected from the samples at the initial diagnosis, which consisted of poorly differentiated adenocarcinoma (Fig. 2). However, because the pathological material at the first diagnosis was obtained by a trans-bronchial lung biopsy, this may reflect only a portion of the disease; 2) ProGRP, a tumor marker of small cell carcinoma, was not elevated at the initial diagnosis; and 3) small cell carcinoma cells, which were first found at autopsy from the pancreas and left kidney specimens, retained the somatic EGFR exon 19 deletion.

In this case, small cell carcinoma cells, detected in the pancreas and left kidney specimens, were confirmed to have retained the somatic EGFR exon 19 deletion and did not have the EGFR T790M mutation, whereas the adenocarcinoma cells, detected in the right kidney autopsy specimen, were confirmed to have retained the somatic EGFR exon 19 deletion and developed the EGFR T790M mutation. This case is noteworthy because the autopsy confirmed different resistant mechanisms, small cell carcinoma transformation from adenocarcinoma and an acquired EGFR T790M mutation, in metastatic lesions in different organs following EGFR-TKI therapy. However, we did not conduct a gene mutation analysis on the squamous cell carcinoma component in the primary tumor at autopsy because the lesion was too small.

Some small cell carcinoma transformation cases have 


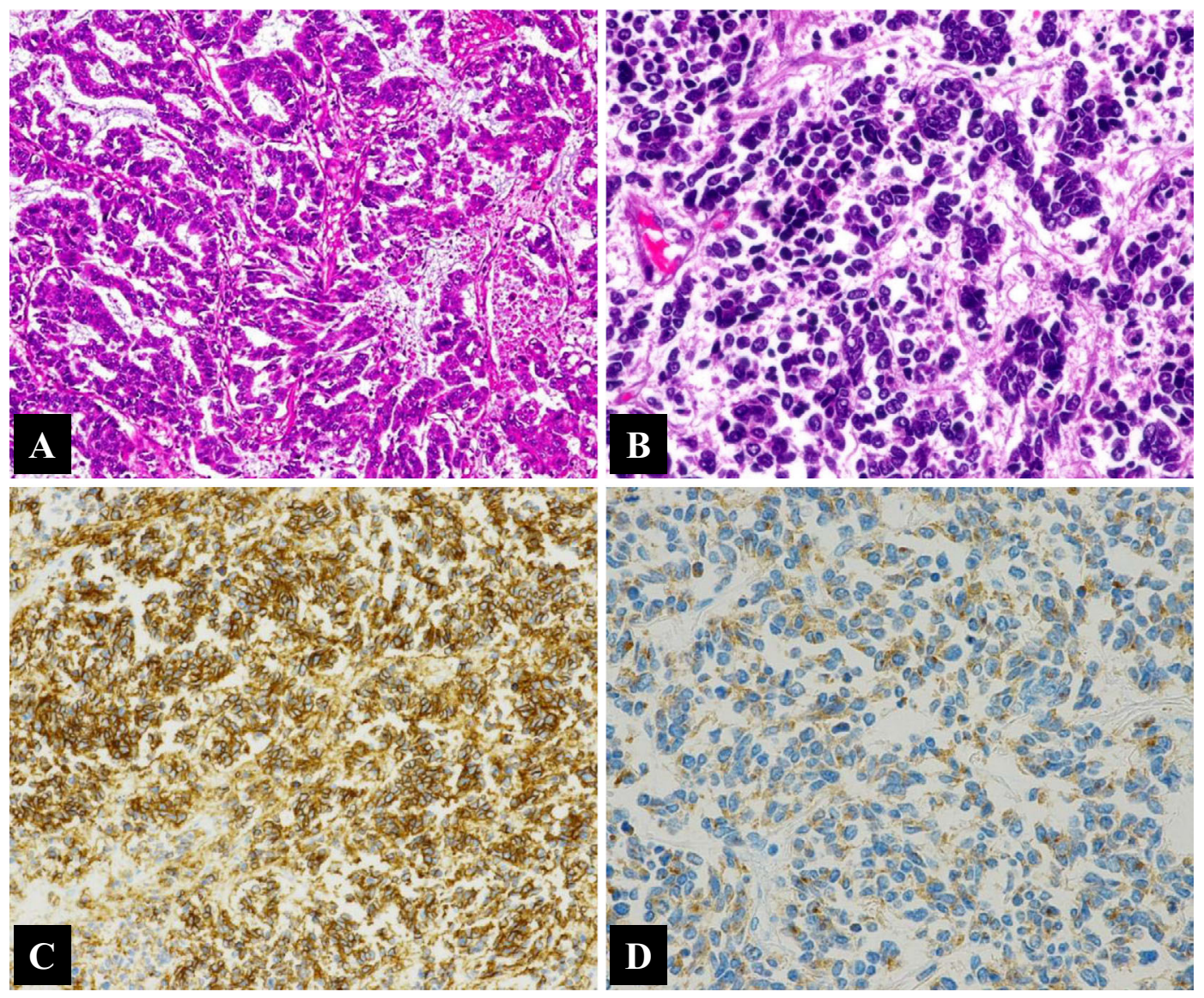

Figure 3. The autopsy specimen in the left upper lung lobe (primary lesion) consisted of poorly differentiated adenocarcinoma (A: $4 \times$ original magnification) and small cell carcinoma (B: $20 \times$ original magnification) with limited squamous cell carcinoma (data not shown). Staining for CD56 (C: 20x original magnification) and synaptophysin (D: 20x original magnification) in the focus of section-B (small cell carcinoma) was positive.

Table. Clinical and Autopsy Diagnosis.

\begin{tabular}{|c|c|c|}
\hline Organ & $\begin{array}{l}\text { Clinical diagnosis } \\
\text { (before EGFR-TKI treatment) }\end{array}$ & $\begin{array}{l}\text { Autopsy diagnosis } \\
\text { (after EGFR-TKI treatment) }\end{array}$ \\
\hline Lung & $\begin{array}{l}\text { Adeno (exon } 19 \text { del., T790M -) } \\
\text { (Fig. 2) }\end{array}$ & $\begin{array}{l}\text { Adeno (Fig. 3A) } \\
\text { Small (Fig. 3B-D) } \\
\text { (Squamous, limited) }\end{array}$ \\
\hline Pancreas & Metastatic lesion on CT (-). & Small (exon 19 del., T790M -) (Fig. 4A) \\
\hline Left kidney & Metastatic lesion on $\mathrm{CT}(-)$. & Small (exon 19 del., T790M -) (Fig. 4B) \\
\hline Right kidney & Metastatic lesion on CT (-). & Adeno (exon 19del., T790M +) (Fig. 4C) \\
\hline $\begin{array}{l}\text { Mediastinum } \\
\text { Epicardium } \\
\text { Liver } \\
\text { Gall bladder } \\
\text { Adrenal gland } \\
\text { Bone marrow } \\
\text { Para-aortic lymph node }\end{array}$ & $\begin{array}{l}\text { Metastatic lesions of mediastinum, } \\
\text { liver, and adrenal gland on CT }(+) \text {. } \\
\text { Biopsy unexamined. }\end{array}$ & Small \\
\hline $\begin{array}{l}\text { Mesentery } \\
\text { Small intestine }\end{array}$ & Metastatic lesions on CT (-). & Small + Adeno \\
\hline $\begin{array}{l}\text { Spleen } \\
\text { Spondylus }\end{array}$ & $\begin{array}{l}\text { Metastatic lesions on CT }(+) \text {. } \\
\text { Biopsy unexamined. }\end{array}$ & Adeno \\
\hline
\end{tabular}

EGFR-TKI: epidermal growth factor receptor-tyrosine kinase inhibitor, Adeno: adenocarcinoma, exon 19 del.: exon 19 deletion, Small: small cell carcinoma, Squamous: squamous cell carcinoma, CT: computed tomography 

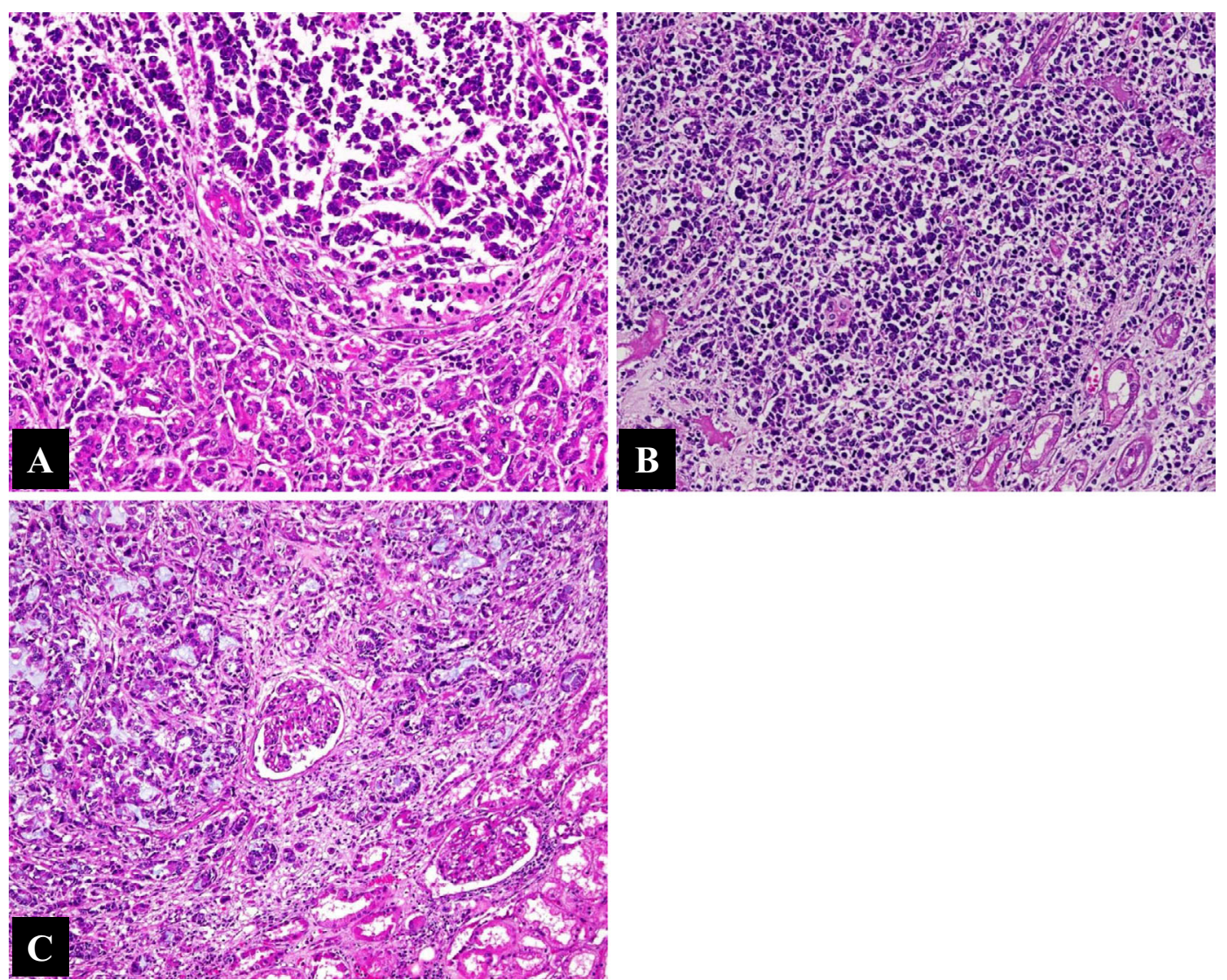

Figure 4. Autopsy specimens reveal small cell carcinoma in the pancreas (A: $4 \times$ original magnification), left kidney metastatic lesions (B: $4 \times$ original magnification) and adenocarcinoma in the right kidney metastatic lesion (C: $4 \times$ original magnification).

been previously reported to be responsive to irinotecan or etoposide $(7,8,11)$. In this case, the patient could not receive additional chemotherapy as his general status rapidly declined after the confirmation of small cell carcinoma transformation. The ProGRP and NSE data from this patient were first confirmed to be elevated just before his death. If his test results had shown elevated ProGRP and NSE levels earlier during the clinical course, then he may have been amenable to chemotherapy for the small cell carcinoma. We therefore recommend the early evaluation of serum ProGRP and NSE levels in patients taking EGFR-TKI at the time of any disease progression.

The causes of transformation to small cell carcinoma are unknown and of great interest. The hypothesis that adenocarcinoma and small cell carcinoma originate from a common uncommitted stem cell has been proposed for a number of years $(8,11,12)$. While, this has not yet been proven, we hope that the clues presented here may help confirm this hypothesis.

In summary, we herein reported an autopsy case of resistance to EGFR-TKI therapy via small cell carcinoma transformation and the EGFR T790M mutation with retained activating EGFR mutation. Further investigation is necessary to clarify the various acquired drug resistance mechanisms in EGFR-mutant lung adenocarcinoma patients.
The authors state that they have no Conflict of Interest (COI).

\section{References}

1. Lynch TJ, Bell DW, Sordella R, et al. Activating mutations in the epidermal growth factor receptor underlying responsiveness of non-small cell lung cancer to gefitinib. N Engl J Med 350: 21292139, 2004.

2. Paez JG, Jänne PA, Lee JC, et al. EGFR mutations in lung cancer: correlation with clinical response to gefitinib therapy. Science 304: 1497-1500, 2004.

3. Kobayashi S, Boggon TJ, Dayaram T, et al. EGFR mutation and resistance of non-small-cell lung cancer to gefitinib. N Engl J Med 352: 786-792, 2005.

4. Pao W, Miller VA, Politi KA, et al. Acquired resistance of lung adenocarcinomas to gefitinib or erlotinib is associated with a second mutation in the EGFR kinase domain. PLoS Med 2: e73, 2005.

5. Engelman JA, Zejnullahu K, Mitsudomi T, et al. MET amplification leads to gefitinib resistance in lung cancer by activating ERBB3 signaling. Science 316: 1039-1043, 2007.

6. Bean J, Brennan C, Shih JY, et al. MET amplification occurs with or without T790M mutations in EGFR mutant lung tumors with acquired resistance to gefitinib or erlotinib. Proc Natl Acad Sci U S A 104: 20932-20937, 2007.

7. Morinaga R, Okamoto I, Furuta K, et al. Sequential occurrence of non-small cell and small cell lung cancer with the same EGFR mutation. Lung Cancer 58: 411-413, 2007.

8. Sequist LV, Waltman BA, Dias-Santagata D, et al. Genotypic and histological evolution of lung cancers acquiring resistance to 
EGFR inhibitors. Sci Transl Med 3: 75ra26, 2011.

9. Alam N, Gustafson KS, Ladanyi M, et al. Small-cell carcinoma with an epidermal growth factor receptor mutation in a never smoker with gefitinib-responsive adenocarcinoma of the lung. Clin Lung Cancer 11: E1-E4, 2010.

10. Yano S, Wang W, Li Q, et al. Hepatocyte growth factor induces gefitinib resistance of lung adenocarcinoma with epidermal growth factor receptor-activating mutations. Cancer Res 68: 9479-9487, 2008.
11. Masaki H, Ishii S, Takeda $Y$, Watanabe $S$, Hojo M, Sugiyama H. A case of lung adenocarcinoma that transformed to small cell carcinoma. Nihon Kokyuuki Gakkaishi (Annals of the Japanese Respiratory Society) 3: 541-547, 2014 (in Japanese, Abstract in English).

12. Fukui T, Tsuta K, Furuta K, et al. Epidermal growth factor receptor mutation status and clinicopathological features of combined small cell carcinoma with adenocarcinoma of the lung. Cancer Sci 98: 1714-1719, 2007.

(C) 2015 The Japanese Society of Internal Medicine http://www.naika.or.jp/imonline/index.html 